\title{
A survey of $M$ stars in the field of view of Kepler space telescope
}

\author{
Mahmoudreza Oshagh ${ }^{1}$, Nader Haghighipour ${ }^{2}$ and Nuno C. Santos ${ }^{1}$ \\ ${ }^{1}$ Centro de Astrofísica, Faculdade de Ciências, Universidade do Porto, Rua das Estrelas, \\ 4150-762 Porto, Portugal \\ email: moshagh@astro.up.pt
}

${ }^{2}$ Institute for Astronomy and NASA Astrobiology Institute, University of Hawaii-Manoa, 2680 Woodlawn Drive, Honolulu, HI 96822,USA

\begin{abstract}
M dwarfs constitute more than $70 \%$ of the stars in the solar neighborhood. They are cooler and smaller than Sun-like stars and have less-massive disks which suggests that planets around these stars are more likely to be Neptune-size or smaller. The transit depths and transit times of planets around M stars are large and well-matched to the Kepler temporal resolution. As a result, $\mathrm{M}$ stars have been of particular interest for searching for planets in both radial velocity and transit photometry surveys. We have recently started a project on searching for possible planet-hosting $\mathrm{M}$ stars in the publicly available data from Kepler space telescope. We have used four criteria, namely, the magnitude, proper motion, $H-K_{s}$ and $J-H$ colors, and searched for M stars in Q0 and Q1 data sets. We have been able to find $108 \mathrm{M}$ stars among which 54 had not been previously identified among Kepler's targets. We discuss the details of our selection process and present the results.
\end{abstract}

Keywords. stars: low-mass, planetary systems

\section{Introduction}

The Kepler space telescope is monitoring more than 150,000 stars in a 105 square degree field of view around the constellations Cygnus and Lyra. The data from this telescope provide a great opportunity for identifying potential terrestrial planets around variety of stars. Among these stars, M dwarfs are ideal targets for searching for terrestrial/habitable planets. These stars have the greatest reflex acceleration due to an orbiting planet, their low surface temperatures place their habitable zones at close distances, and their light curves show large decrease when they are transited by a planet. The transit depths and transit times of planets around M stars are also large and well-matched to the Kepler temporal resolution. We have surveyed Kepler's publicly available data from quarters Q0 and Q1, and identified more than a hundred M stars. In this paper, we discuss our methodology and present our selection process.

\section{Selection Criteria}

Traub \& Cutri (2008) were the first to make an attempt to identify M stars in Kepler's field of view. They considered stars with $K_{s}$ smaller than $13.5 \mathrm{mag}$, and proposed a selection process based on a criterion on $H-K_{s}$ vs. $J-H$ colors. Since M stars are relatively faint and abundant, Traub \& Cutri (2008) suggested that any detected M star is likely to be nearby and therefore likely to have a relatively large proper motion. Using these criteria, these authors estimated that close to $1600 \mathrm{M}$ stars should exist in Kepler's field of view. A recent article by Batalha et al. (2010) suggests that the actual number of M stars in Kepler's field of view may be twice as large. Using selection criteria based on 
Table 1. Star Counts as a Function of Effective Temperature and Magnitude

\begin{tabular}{cccc}
\hline Mag & $\begin{array}{c}T_{\text {eff }} \\
(4500-5500)\end{array}$ & $\begin{array}{c}T_{\text {eff }} \\
(3500-4500)\end{array}$ & Total \\
\hline $6<K<7$ & 0 & 0 & 0 \\
$7<K<8$ & 0 & 0 & 0 \\
$8<K<9$ & 0 & 5 & 5 \\
$9<K<10$ & 1 & 3 & 4 \\
$10<K<11$ & 0 & 9 & 9 \\
$11<K<12$ & 0 & 13 & 13 \\
$12<K<13$ & 1 & 13 & 14 \\
$13<K<14$ & 0 & 1 & 1 \\
$14<K<15$ & 0 & 0 & 0 \\
\hline
\end{tabular}

a star's surface gravity, effective temperature, and inferred stellar radius, these authors were able to show that approximately $3000 \mathrm{M}$ stars are present in the field of view of Kepler.

We used the following criteria to look for M stars in Kepler's publicly available data of quarters Q0 and Q1. These criteria are somewhat similar to those of Traub \& Cutri (2008) with the exception that we expanded the requirements on the magnitudes of stars using the ranges suggested by Leggett (1992):

- $K$ magnitude equal to or smaller than $15 \mathrm{mag}$,

- $J-H$ color in the range of 0.42 to 0.78 ,

- $H$ - $K$ color in the range of 0.12 to 0.50 ,

- Proper motion bigger than 0.1 .

Our search resulted in identifying $108 \mathrm{M}$ stars. Among these stars, 27 are in both the Q0 and Q1 data sets. When comparing with the sample of M stars presented by Batalha et al. (2010), we discovered that there are no data on the effective temperature and surface gravity of 54 of our M stars. As a result, these $54 \mathrm{M}$ stars were not identified by Batalha et al. (2010). One example of these M stars is LHS 6343, a member of a M star binary system with a transiting brown dwarf (Johnson et al. 2011). Table 1 shows 46 of these $54 \mathrm{M}$ stars that have $\log g>3.5$. These stars have been binned by magnitude and effective temperature.

This work has been supported by the European Research Council/European Community under the FP7 through a Starting Grant, as well as in the form of grant reference PTDC/CTEAST/098528/2008 funded by Fundação para a Ciência e a Tecnologia (FCT), Portugal. NCS would further like to thank the support from FCT through a Ciência , 2007 contract funded by FCT/MCTES (Portugal) and POPH/FSE (EC). NH acknowledges support from NASA Astrobiology Institute (NAI) under Cooperative Agreement NNA04CC08A at the Institute for Astronomy, University of Hawaii, NAI central, and NASA EXOB grant NNX09AN05G.

\section{References}

Batalha, N. M., et al. 2010, ApJ, 713, L109

Johnson, J. A., et al. 2011, ApJ, 730, id.79

Leggett, S. K. 1992, ApJS, 82, 351

Traub, W. \& Cutri, R. 2008, in: Extreme Solar Systems, Eds. D. Fischer, F. A. Rasio, S. E., Thorsett, \& A. Wolszczan, ASP Conf. Ser., 398, 475 